\title{
Using Jaccard Distance Measure for Unsupervised Activity Recognition with Smartphone Accelerometers
}

\author{
Xianlong Wang ${ }^{1}$, Yonggang $\mathrm{Lu}^{1, *}$, Dachuan Wang ${ }^{1}, \mathrm{Li} \mathrm{Liu}^{2, *}$, and Huiyu Zhou ${ }^{3}$ \\ ${ }^{1}$ School of Information Science and Engineering, Lanzhou University, Lanzhou, Gansu 730000, \\ China. \\ \{ylu@lzu.edu.cn,910379526@qq.com, 1781368972@qq.com\} \\ ${ }^{2}$ School of Software Engineering, Chongqing University, Chongqing 400044, China. \\ \{dcsliuli@cqu.edu.cn\} \\ ${ }^{3}$ Institute of Electronics, Communications and Information Technology, Queens \\ University of Belfast, Belfast, BT3 9DT, United Kingdom. \\ \{h.zhou@ecit.qub.ac.uk\} \\ *Corresponding authors: ylu@lzu.edu.cn, dcsliuli@cqu.edu.cn
}

\begin{abstract}
The rapid popularity of smartphones has led to a growing research interest in human activity recognition (HAR) with the mobile devices. Accelerometer is the most commonly used sensor of smartphone for HAR. Most supervised HAR methods have been developed. However, it is very difficult to collect the annotated or labeled training data for HAR. So, developing of effective unsupervised methods for HAR is very necessary. The accuracy of the unsupervised activity recognition can be greatly affected by feature extraction methods and distance measures. Although Euclidean distance measure is commonly used in activity recognition, it is not suitable for measuring distance when the number of features is very large, which is usually the case in HAR. Jaccard distance is a distance measure based on mutual information theory and can better represent the differences between nonnegative feature vectors than Euclidean distance. In this work, the Jaccard distance measure is applied to HAR for the first time. In the experiments, the results of the Jaccard distance measure and the Eucildean distance measure are compared, using three different feature extraction methods. Two different evaluation methods are used to comprehensively analyze the final results: (a) C-Index before clustering, (b) FM-index after using five different clustering methods which are Spectral Cluster, Single-Linkage, Ward-Linkage, Average-Linkage, and K-Medoids. Experiments show that, almost for every combination of the feature extraction methods and the evaluation methods, the Jaccard distance is consistently better than the Euclidean distance for unsupervised HAR.
\end{abstract}

Keywords: Activity Recognition; Jaccard Distance; Feature Extraction; Unsupervised HAR; Smartphone

\section{Introduction}

With the improvement of health awareness of people and the continuous development of science technology, HAR is increasingly used in intelligent life, motion detection, diagnosis of chronic diseases and healthy field $[1,2,3,4,5]$. The health care system can keep track of the health of user, and capture the activity of user for a long-term, which can help the doctor to diagnose whether the user suffers from chronic diseases or not, and to urge the user to take proper exercise every day. The specialized medical 
equipment, wearable sensors or camera-based computer vision system have been used to recognize human activities, but they all require complex equipment and require camera to be placed in a fixed position, which is inconvenient for daily activity recognition.

Alternatively, smartphone is very popular now and people can carry it anytime, anywhere. Most smartphones are equipped with a rich set of embedded sensors, such as accelerometer, GPS sensors, gyroscopes and so on. So smartphone has become an active field of research in the domain of perception and mobile computing.

Within various sensors of smartphone, accelerometer is the most commonly used sensor for recording human activity signals. A. M. Khan et al. [6] have used a smartphone with a built-in triaxial accelerometer to collect five daily physical activities from five body positions, and the average classification accuracy of their method is about $96 \%$. In the work of Yi He et al. [7], three different sensors including accelerometer, gyroscope, and magnetic sensor embedded in a smartphone placed at the chest of a subject are used for HAR. The ten highest-ranked features are selected from one hundred and forty features extracted from the sensors. Then, a hierarchical classifier system is used to recognize fifteen activities. They get a classification accuracy of $95.03 \%$. Their work also shows that within the three embedded sensors, the accelerometer is the most significant sensor for activity recognition.

Usually, raw time series data cannot be applied on HAR directly, feature extraction methods have to been used to produce a new data representation (called features) from the raw acceleration data before the classification.

Popular features extracted from the acceleration data in the previous work can be divided into two types: time-domain features and frequency-domain features. Time-domain features include mean, variance, standard deviation, etc., while frequency-domain features include frequency-domain entropy, discrete FFT coefficients, etc. $[8,15,16]$. In this work, besides frequency-domain features and time-domain features, the mixed-domain features which include features from both time-domain and frequency-domain are also used to systematically study the effects of feature extraction for HAR.

In the traditional approaches to HAR, standard supervised classification methods, such as Support Vector Machine (SVM), K-Nearest Neighbor (KNN), Decision Tree, etc., have been used after feature extraction. Lin Sun et al. [9] have used accelerometer embedded mobile phones to monitor seven common physical activities of seven subjects and have boosted the overall accuracy from $91.5 \%$ to 93.1\% by improving the SVM method. Alvina Anjum et al. [10] have collected a dataset of seven different activities, which include walking, running, ascending stairs, descending stairs, cycling, driving and remaining inactive using cell phone sensors and have evaluated a number of classification methods including Naive Bayes, Decision Tree, KNN and SVM. The Decision Tree classifier outperforms the other classifiers, and on average it produces a true positive rate of $95.2 \%$.

All the above studies are using supervised classification methods to perform activity recognition. For the supervised methods, a large number of annotated data are required to train the classifiers. However, the data annotation is a difficult task which usually requires lots of time and efforts. So the unsupervised methods which can use the raw data directly for HAR have special advantages. In our previous work [11], an unsupervised method is proposed for HAR with time-domain features extracted from the data collected using smartphone accelerometers. It is shown that the unsupervised method is also effective to distinguish several daily activities. The unsupervised methods usually classify data based on the similarities between the data points. So the accuracy of the unsupervised classification methods can be greatly affected by the distance measure (or similarity measure) applied on the feature vectors. Although Euclidean distance measure is most commonly used, it may fail to measure the 
differences between features effectively in the high-dimensional space [12]. Jaccard distance [13] is a distance measure based on mutual information theory and can better represent the differences between nonnegative feature vectors than Euclidean distance. In this work, the Jaccard distance measure is applied to HAR for the first time and its superiority to the Euclidean distance measure is shown by the experiments.

\section{Related work}

Recently, position and orientation independent activity recognition using smartphone accelerometers has also been investigated. Lin Fan et al. [14] use resultant acceleration to eliminate the effects of the phone orientations. A feature set including seven time-domain features and three frequency-domain features is used for classifying five activities: staying still, walking, jogging, ascending stairs and descending stairs. The classification accuracy produced by their method is $80.29 \%$. Fen Miao et al. [15] have used accelerometer, gyroscope, proximity sensor, light sensor and magnetic sensor of a smartphone for HAR. The magnitude of linear acceleration combined with signals collected from gyroscope sensor and magnetic sensor are used. Then six time-domain statistical features are extracted for recognizing five typical physical activities which include staying still, walking, jogging, ascending stairs and descending stairs. Their results show the possibility of position and orientation independent HAR using smartphones without firm attachment to the body. In this work, orientation-independent HAR is done by using the resultant acceleration data from the smartphones.

Generally, the accuracy of the unsupervised HAR is affected by feature extraction methods and distance measures. In the work of Fen Miao et al. [15], the time-domain features, including mean, standard deviation, median, skewness, kurtosis, and Inter-quartile-range are extracted from the resultant acceleration data to study the six daily activities using Decision Tree, Naive Bayes and an improved SVM. In the work of Carina Figueira et al. [16], the activities of standing, sitting, walking, running, ascending stairs and descending stairs are studied. They have extracted mixed-domain features, including root mean square, median absolute deviation, standard deviation, spectral roll on, mean power spectrum, max power spectrum from the data. In this work, three feature extraction methods based on frequency-domain, time-domain and mixed-domain are compared for HAR.

Many clustering methods have been applied on unsupervised HAR. In the work of Inês P. Machado et al. [17], the activities of standing, sitting, walking, running, lying are studied with mixed-domain features. Four clustering methods, including K-Means, Spectral Clustering, Mean Shift and Affinity Propagation (AP) based on Euclidean distance are used to distinguish different activities. In the subject-independent context, the accuracy rate of K-Means clustering reaches $88.75 \%$, while the accuracy rate of other clustering algorithms ranges from $30 \%$ to $45 \%$. In the case of subject-dependent context, the accuracy rate of K-Means clustering reaches $99.29 \%$ and the accuracy of other clustering methods ranges from 53\% to 93.96\%. In work of Gomes, A. L. G. N. [18], K-Means, Mini Batch K-Means, AP, Mean Shift, DBSCAN, Spectral Clustering, and Ward-Linkage have been applied for HAR. They have found that the Spectral Clustering has produced the best results with an accuracy rate of $89.1 \% \pm 8.8 \%$. Amitha. $\mathrm{R}$ et al. [19] have proposed a system using Naive Bayes classifier combined with a hierarchical agglomerative clustering algorithm for activity tracking. Hierarchical agglomerative clustering is a "bottom up" approach based on the similarity or distance of feature vectors. Different in the definition of the similarity between different clusters, there are four commonly used hierarchical clustering methods: Signal-Linkage, Complete-Linkage, Average-Linkage and Ward-Linkage. 
In this work, Spectral Clustering, Single-Linkage, Ward-Linkage, Average-Linkage and K-Medoids are used for HAR.

Euclidean distance measure is commonly used in the clustering algorithms to measure the distances between the feature vectors. However, Euclidean distance metric usually cannot measure the differences effectively between features in high-dimensional space due to the curse of dimensionality [20]. The normalization of features is also needed for using the Euclidean distance measure. The drawbacks of the Euclidean distance measure can be avoided by using the Jaccard distance measure instead [21]. Jaccard distance is a distance measure based on mutual information theory. It measures the degree of the overlap between two sets of nonnegative feature values, so the normalization of features is not needed, and the distance is less affected by the number of the features than the Euclidean distance. As far as we know, the Jaccard distance measure is applied to HAR for the first time in this work.

\section{Method}

\subsection{Data Collection}

In experiments, both an UCI dataset and a dataset collected by ourselves are used for HAR. The UCI dataset includes triaxial acceleration data of walking, running, ascending stairs, descending stairs, sitting and standing of 30 subjects [22]. However, the data of 13 subjects have missing values or invalid values. So only the data of the rest 17 subjects are used in our experiments.

The dataset collected by ourselves are from 6 healthy volunteers with ages from 22 to 28 . Each of them was instructed to carry a Samsung Galaxy SIII smartphone in their front pants pocket while performing a specific set of activities for a certain time. These activities are walking, jogging, ascending stairs, descending stairs, sitting and standing. The acceleration data with a sampling frequency of $50 \mathrm{~Hz}$ are collected.

\subsection{Feature Extraction}

Before feature extraction, to reduce the bias caused by sensor sensitivity and noise, a sliding window with $50 \%$ overlap is employed to divide the time series into smaller time windows. For the UCI dataset the window of 2.56 seconds is used, while for our dataset, the window of 5.12 seconds is used. Similar as previous works [23], the magnitude of the acceleration, $A_{3 a}$, is used for feature extraction, which is insensitive to the orientation and position of the devices. $A_{3 a}$ can be represented as:

$$
A_{3 a}=\sqrt{A_{x}^{2}+A_{y}^{2}+A_{z}^{2}}
$$

The magnitudes of the acceleration date within each sliding window are then used for feature extraction. Three feature extraction methods based on frequency-domain, time-domain and mixed-domain are used in the experiments, which are explained in detail below.

\section{(a)Time-domain Feature Extraction}

The same time-domain features used in the work of Fen Miao et al. [15] are extracted, which includes Mean, Standard Deviation, Median, Skewness, Kurtosis, and Inter-Quartile-Range. 


\section{(b) Mixed-domain Feature Extraction}

The same mixed-domain feature set as reported in the work of Carina Figueira et al. [16] are extracted, which includes Root Mean Square(RMS), Median Absolute Deviation(MAD), Standard Deviation, Spectral Roll On, Mean Power Spectrum, Max power Spectrum.

\section{(c) Frequency-domain Feature Extraction}

In the frequency-domain, to eliminate the effect of edge samples, a sine window is first multiplied to the data in the window. The sine window is defined as:

$$
\operatorname{win}=\sin ((i-1) \pi /(\text { winLen }-1))^{2} \quad i \in[1 \text { : winLen }]
$$

Where winLen represents the length of the window.

Then FFT is applied to the data of each window. In order to improve the frequency resolution and reduce spectral leakage, the magnitudes of the Fourier coefficients are convolved with a hamming window of size 5 . Then the final results of each window are used as feature vectors.

\subsection{Jaccard Distance Measure}

In this work, Jaccard distance [13] is used to calculate the similarity between feature vectors. If $\mathrm{X}=(\mathrm{x} 1$, $\mathrm{x} 2, \ldots, \mathrm{xn})$ and $\mathrm{Y}=(\mathrm{y} 1, \mathrm{y} 2, \ldots, \mathrm{yn})$ are two vectors and all $x i, y i(1 \leq \mathrm{i} \leq \mathrm{n})$ are non-negative, their Jaccard similarity coefficient is defined as:

$$
J(X, Y)=\frac{\sum_{i=1}^{n} \min (x(i), y(i))}{\sum_{i=1}^{n} \max (x(i), y(i))}
$$

and their Jaccard distance is defined as:

$$
D_{J}(X, Y)=1-J(X, Y) .
$$

The value of the Jaccard distance ranges from 0 to 1 , where 1 represents the identical sets, while 0 represents the disjoint sets. Because in our work, all the feature values extracted using the three feature extraction methods are greater than or equal to 0 , Jaccard distance can be readily applied to them.

\section{Experiments}

\subsection{Design of the Experiments}

To compare the effectiveness of the Euclidean distance and the Jaccard distance in unsupervised HAR, the following procedures of the experiments are executed (as shown in Fig. 1):

(a) Collect the acceleration data of walking, jogging, ascending stairs, descending stairs, standing and sitting;

(b) Divide the time series data into small time windows using a sliding window with $50 \%$ overlap; 
(c) Use the three feature extraction methods to extract features based on the magnitude of the acceleration;

(d) Calculate the distance between the feature vectors using both the Jaccard distance and Euclidean distance;

(e) Use C-index to compare Jaccard distance and Euclidean distance;

(f) Cluster the data based on the distances between feature vectors using the five different clustering methods which include Spectral Cluster, Single-Linkage, Ward-Linkage, Average-Linkage, and K-Medoids, and then measure the clustering results using FM-index.

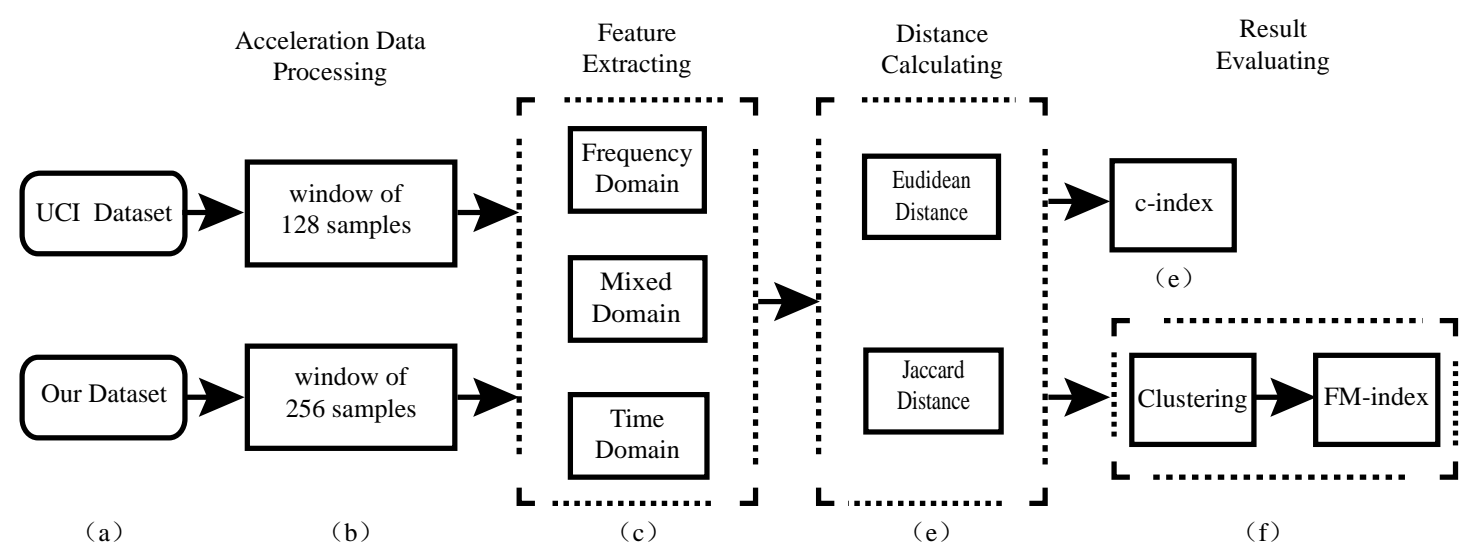

Fig. 1. Procedure of Experiments.

\subsection{Evaluation Criterion}

Both C-index [24] and FM-index [25] are used to evaluate the experimental results. C-index is used to measure the compatibility of the distance measure with the actual class labels. A smaller C-index value indicates a better compatibility where the data points have smaller distances within the same class and greater distances between different classes. The C-index is defined as:

$$
C=\frac{S-S_{\min }}{S_{\max }-S_{\min }}
$$

where $S$ is the sum of the distances over all $m$ pairs of objects from the same class, $S_{\min }$ is the sum of the $m$ smallest distances if all pairs of objects are considered. $S_{\max }$ is the sum of the $m$ largest distances out of all the pairs. The interval of the C-index values is between 0 and 1.

The FM-index is used to evaluate the clustering results produced by a clustering method. The maximum value of the FM-index is 1 , which means that the clustering result are the same as the actual result, while the minimum value is 0 , which means the clustering result and actual result are completely different. For FM-index, a larger value means a better similarity between the clustering result and the actual result. 


\section{Result}

For the UCI dataset containing 17 subjects and our dataset containing 6 subjects, the C-index is used to measure the distance generated by three different feature extraction methods and the two different distance measures. The results are shown in Table 1.

Table 1. Comparison of different feature extraction methods and distance measures on UCI dataset and our dataset using C-index.

\begin{tabular}{|c|c|c|c|c|c|c|}
\hline \multirow[t]{2}{*}{ Dataset } & \multicolumn{2}{|c|}{$\begin{array}{c}\text { Frequency } \\
\text { Domain } \\
\end{array}$} & \multicolumn{2}{|c|}{$\begin{array}{c}\text { Time } \\
\text { Domain } \\
\end{array}$} & \multicolumn{2}{|c|}{$\begin{array}{c}\text { Mixed } \\
\text { Domain } \\
\end{array}$} \\
\hline & Euclidean & Jaccard & Euclidean & Jaccard & Euclidean & Jaccard \\
\hline \multirow{17}{*}{$\begin{array}{c}\text { UCI } \\
\text { Dataset }\end{array}$} & 0.0645 & 0.0298 & 0.1723 & 0.0621 & 0.1127 & 0.1087 \\
\hline & 0.0445 & 0.0310 & 0.1105 & 0.0375 & 0.1058 & 0.0889 \\
\hline & 0.0439 & 0.0402 & 0.1596 & 0.0605 & 0.0932 & 0.0865 \\
\hline & 0.0310 & 0.0892 & 0.2715 & 0.1181 & 0.1093 & 0.1053 \\
\hline & 0.0617 & 0.0554 & 0.1470 & 0.0650 & 0.0931 & 0.0941 \\
\hline & 0.2034 & 0.0285 & 0.1488 & 0.0691 & 0.1383 & 0.0987 \\
\hline & 0.0555 & 0.0404 & 0.2497 & 0.0712 & 0.1632 & 0.1416 \\
\hline & 0.0606 & 0.0281 & 0.1274 & 0.0438 & 0.0608 & 0.0568 \\
\hline & 0.0531 & 0.0373 & 0.2003 & 0.0694 & 0.0973 & 0.0849 \\
\hline & 0.0406 & 0.0310 & 0.1504 & 0.0412 & 0.1072 & 0.0809 \\
\hline & 0.0577 & 0.0644 & 0.0789 & 0.0408 & 0.0409 & 0.0432 \\
\hline & 0.0885 & 0.0116 & 0.0927 & 0.0594 & 0.0583 & 0.0421 \\
\hline & 0.1229 & 0.1316 & 0.2175 & 0.0849 & 0.1810 & 0.1707 \\
\hline & 0.0608 & 0.0311 & 0.0770 & 0.0256 & 0.0256 & 0.0245 \\
\hline & 0.0586 & 0.0254 & 0.1850 & 0.0674 & 0.1688 & 0.1379 \\
\hline & 0.0745 & 0.0299 & 0.1779 & 0.0857 & 0.1356 & 0.1279 \\
\hline & 0.0395 & 0.0287 & 0.1367 & 0.0444 & 0.0581 & 0.0624 \\
\hline \multirow{6}{*}{$\begin{array}{c}\text { Our } \\
\text { Dataset }\end{array}$} & 0.0294 & 0.0282 & 0.1734 & 0.0049 & 0.0525 & 0.0335 \\
\hline & 0.0314 & 0.0303 & 0.0803 & 0.0120 & 0.0378 & 0.0272 \\
\hline & 0.0296 & 0.0239 & 0.0401 & 0.0047 & 0.0219 & 0.0169 \\
\hline & 0.0270 & 0.0253 & 0.1290 & 0.0097 & 0.0702 & 0.0650 \\
\hline & 0.0201 & 0.0210 & 0.1652 & 0.0155 & 0.0606 & 0.0421 \\
\hline & 0.0286 & 0.0273 & 0.0472 & 0.0082 & 0.0227 & 0.0197 \\
\hline
\end{tabular}

From Table 1, it can be seen that most of the C-index produced using the Jaccard distance measure are better than that produced using the Euclidean distance, regardless of which feature extraction methods are used. Within 69 results, there are only 6 exceptions. The results show that the Jaccard distance measure can measure the distances between feature vectors better than the Euclidean distance measure. Based on the distances computed using the two distance measures, five different clustering methods which include Spectral Cluster, Single-Linkage, Ward-Linkage, Average-Linkage, and K-Medoids are used to cluster the dataset. The FM-index is then used to measure the clustering results. Table 2 shows the FM-indices produced using different combinations of the three feature extraction methods, the five clustering methods and the two distance measures on the UCI dataset and our dataset, where the FM-indices of all the subjects in each dataset are averaged. 
Table 2. Comparison of different feature extraction methods, distance measures and clustering methods using FM-index.

\begin{tabular}{|c|c|c|c|c|c|c|c|}
\hline \multirow[t]{2}{*}{ Dataset } & \multirow{2}{*}{$\begin{array}{c}\text { Clustering } \\
\text { method }\end{array}$} & \multicolumn{2}{|c|}{$\begin{array}{c}\text { Frequency } \\
\text { Domain }\end{array}$} & \multicolumn{2}{|c|}{$\begin{array}{c}\text { Time } \\
\text { Domain }\end{array}$} & \multicolumn{2}{|c|}{$\begin{array}{c}\text { Mixed } \\
\text { Domain }\end{array}$} \\
\hline & & Euclidean & Jaccard & Euclidean & Jaccard & Euclidean & Jaccard \\
\hline \multirow{5}{*}{$\begin{array}{c}\text { UCI } \\
\text { dataset }\end{array}$} & $\begin{array}{l}\text { Spectral } \\
\text { clustering }\end{array}$ & 0.543 & 0.583 & 0.344 & 0.378 & 0.387 & 0.433 \\
\hline & $\begin{array}{c}\text { Single } \\
\text { Linkage }\end{array}$ & 0.807 & 0.851 & 0.723 & 0.771 & 0.612 & 0.680 \\
\hline & $\begin{array}{c}\text { Ward } \\
\text { Linkage }\end{array}$ & 0.770 & 0.810 & 0.763 & 0.791 & 0.635 & 0.664 \\
\hline & $\begin{array}{l}\text { Average } \\
\text { Linkage }\end{array}$ & 0.790 & 0.871 & 0.757 & 0.814 & 0.618 & 0.687 \\
\hline & K-Medoids & 0.653 & 0.654 & 0.633 & 0.645 & 0.589 & 0.604 \\
\hline \multirow{5}{*}{$\begin{array}{c}\text { Our } \\
\text { dataset }\end{array}$} & $\begin{array}{l}\text { Spectral } \\
\text { clustering }\end{array}$ & 0.674 & 0.688 & 0.543 & 0.582 & 0.387 & 0.442 \\
\hline & $\begin{array}{c}\text { Single } \\
\text { Linkage }\end{array}$ & 0.845 & 0.895 & 0.878 & 0.904 & 0.620 & 0.658 \\
\hline & $\begin{array}{c}\text { Ward } \\
\text { Linkage }\end{array}$ & 0.895 & 0.910 & 0.887 & 0.909 & 0.652 & 0.701 \\
\hline & $\begin{array}{l}\text { Average } \\
\text { Linkage }\end{array}$ & 0.869 & 0.886 & 0.836 & 0.870 & 0.636 & 0.695 \\
\hline & K-Medoids & 0.749 & 0.729 & 0.739 & 0.726 & 0.620 & 0.624 \\
\hline
\end{tabular}

It can be seen from Table 2 that, regardless of which combination of the feature extraction methods and clustering methods are used, the FM-indices produced using the Jaccard distance measure are consistently better than the results produced using the Euclidean distance measure. The results also show the superiority of the Jaccard distance measure over the Euclidean distance measure for distinguishing different activities. It can also be seen in Table 2 that the FM-indices produced using the frequency-domain feature extraction method are all better than these produced using the other two feature extraction methods, except the result of using the Single-Linkage on our dataset. This indicates that the frequency-domain features are more effective than the other two features for unsupervised HAR.

To further compare the Jaccard distance measure and the Euclidean distance measure for activity recognition, the FM-indices produced using the five clustering methods are averaged for each combination of the distance measure and the feature extraction methods. The results are shown in Fig. 2. It can be seen that the FM-index produced using Jaccard distance is better than that produced using Euclidean distance regardless of which feature extraction methods are used, while the FM-index produced using the frequency-domain feature extraction method is better than that produced using the other two feature extraction methods. 


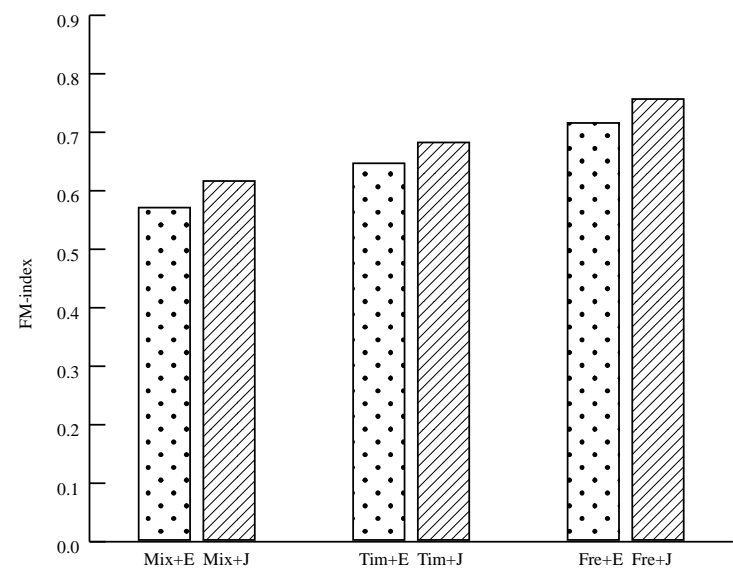

(a)

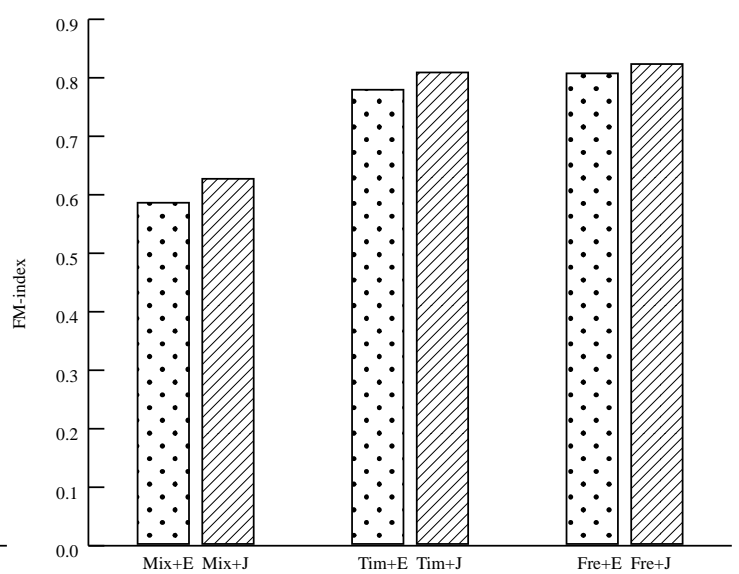

(b)

Fig. 2. Comparison of different combinations of the feature extraction methods and the distance measures using the average of FM-index of the five clustering methods on (a) UCI dataset, and (b) our dataset, where "Fre", “Tim”, “Mix” represent frequency-domain, time-domain, mixed-domain feature extraction methods respectively, and “E”, “J” represent Euclidean distance and Jaccard distance respectively.

\section{Conclusion}

In this paper, the Jaccard distance measure is proposed to replace the Euclidean distance measure for unsupervised HAR. Both the C-index before clustering and the FM-index after clustering show the superiority of the Jaccard distance measure over the Euclidean distance measure for unsupervised HAR. It is also found that the frequency-domain feature extraction method is better than the time-domain feature extraction method and the mixed-domain feature extraction method for unsupervised HAR using the proposed method. Future work includes applying the Jaccard distance measure on more datasets and improving the feature extraction methods.

\section{Acknowledgments}

This work is supported by the National Science Foundation of China (Grants No. 61272213) and the Fundamental Research Funds for the Central Universities (Grants No. lzujbky-2016-k07).

\section{References}

1. Nie, L., Akbari, M., Li, T., Chua, TS. (2014): A joint local-global approach for medical terminology assignment. In: Medical Information Retrieval Workshop at SIGIR 2014. p. 24

2. Nie, L., Wang, M., Zhang, L., Yan, S., Bo, Z., Chua, TS. (2014): Disease inference from health-related questions via sparse deep learning. IEEE Trans Knowl Data Eng 27(8):2107-2119

3. Nie, L., Wang, M., Zhang, L., Yan, S., Bo, Z., Chua, TS. (2014): Disease inference from health-related questions via sparse deep learning. IEEE Trans Knowl Data Eng 27(8):2107-2119

4. Liu, L., Peng, Y., Liu, M., Huang, Z. (2015): Sensor-based human activity recognition system with a multilayered model using time series shapelets. Knowl-Based Syst 90:138-152 26. Nie L, Zhang L, Yang Y, Wang M (2015) Hong, Richang, C.T.S.: Beyond doctors: Future health prediction from multimedia and 
multimodal observations. In: Proceedings of the 2015 ACM conference on Multimedia. pp. 1-9. ACM

5. Pitta F, Takaki MY, Oliveira NHd, Sant'Anna TJP, Fontana AD, Kovelis D, et al.: Relationship between pulmonary function and physical activity in daily life in patients with COPD. In: Respir Med. 2008;102:1203-7

6. Khan, A. M., Lee, Y. K., Lee, S. Y., \& Kim, T. S. (2010).: Human Activity Recognition via an Accelerometer-Enabled-Smartphone Using Kernel Discriminant Analysis. In: International Conference on Future Information Technology (pp.1-6). IEEE.

7. He, Y., Li, Y.: Physical activity recognition utilizing the built-in kinematic sensors of a smartphone.In: International Journal of Distributed Sensor Networks, 481580 (2013)

8. Huynh, T., Schiele, B.: Analyzing features for activity recognition. In: Proceedings Conference on Smart Objects and Ambient Intelligence. ACM, New York, 159C163 (2005)

9. Sun, L., Zhang, D., Li, B., Guo, B., Li, S. (2010): Activity recognition on an accelerometer embedded mobile phone with varying positions and orientations. In: Lect Notes Comput Sci 6406:548-562

10. Anjum, A. and Ilyas, A.: Activity recognition using smartphone sensors. In: 2013 IEEE Consumer Communications and Networking Conference (CCNC), Jan. 2013, pp. 914-919

11. Lu, Y., Wei, Y., Liu, L., Zhong, J., Sun, L. and Liu, Y.: Towards unsupervised physical activity recognition using smartphone accelerometers, pp. 1-19, 2016

12. Beyer, K.; Goldstein, J.; Ramakrishnan, R.; Shaft, U. (1999): When is "Nearest Neighbor" Meaningful?. In: Proc. 7th International Conference on Database Theory - ICDT'99. LNCS. 1540: 217-235

13. Michael, L., David, W. (1971): Distance between sets. In: Nature, 234 (5): 34-35

14. Fan, L., Wang, J. and Wang, H.: Activity recognition based on position-independent smartphone. In: Journal of Computational Information Systems, vol. 10, no. 11, pp. 4921-4928, 2014

15. Miao, F., He, Y., Liu, J., Li, Y.: Identify Typical Physical Activity on Smartphone with Varying Positions and Orientations. In: Biomed. Eng. Online 2015, 14, 32

16. Figueira, C., Ricardo,M. and Hugo, G.:Body Location Independent Activity Monitoring. In: BIOSIGNALS (2016)

17. Machado, I.P., Gomes, A.L., Gamboa, H., Paixão, V., Costa, R.M.: Human activity data discovery from triaxial accelerometer sensor: non-supervised learning sensitivity to feature extraction parameterization. In: Inf Process Manage. 2015;51:204-14

18. Gomes, A. L. G. N. (2014).: Human activity recognition with accelerometry: novel time and frequency features. Nov-2014.

19. Amitha, R. and K, Rajakumari.: Hierarchical Clustering Based Activity Tracking System in a Smart Environment. In: Intenational Journal of Comptuer Science and technology, September 2012, IJCST, Vol. 3. 2229-4333

20. Richard E.B., Rand Corporation.: Dynamic programming. In: Princeton University Press. 1957. ISBN 978-0-691-07951-6

21. Jaccard, P. (1901): Distribution de la flore alpine dans le Bassin des Dranses et dans quelques regions voisines. In: Bulletin de la Société Vaudoise des Sciences Naturelles, 37, 241-272

22. https://archive.ics.uci.edu/ml/datasets/Human+Activity+Recognition+Using+Smartphones

23. Wang, D., Liu, L., Wang, X., Lu, Y.: A Novel Feature Extraction Method on Activity Recognition Using Smartphone. In: Web-Age Information Management: WAIM 2016 International Workshops, MWDA, SDMMW, and SemiBDMA, Nanchang, China, June 3-5, 2016, Revised Selected Papers. S. Song and Y. Tong. Cham, Springer International Publishing: 67-76 
24. Hubert, L. and Schultz, J.: Quadratic assignment as a general data-analysis strategy. In: British Journal of Mathematical and Statistical Psychology 29 (1976), 190-241.

25. Fowlkes, E., Mallows, C.: A method for comparing two hierarchical clusterings. In: Journal of the American Statistical Association 78(383), 553-569 (1983) 